\title{
The Effect Mechanism of Leader's Harmonious Orientation on Employees' Innovation Behavior: Based on Mediating Effect of Employees' Prosocial Motivation
}

\author{
Chao-Wei WANG ${ }^{1, a, *}$, Hui JIN ${ }^{2, b}$, Hao-Bin $\mathrm{Xia}^{3, \mathrm{c}}$ \\ ${ }^{1,2,3}$ School of Economics and Management, Jiangsu University of Science and Technology, Zhenjiang, Jiangsu, China \\ a1766125117@qq.com, ${ }^{b} 48051382 @ q q . c o m,{ }^{c} 530129036 @ q q . c o m$ \\ *Corresponding author
}

\begin{abstract}
Based on the local cultural background, this research introduces employees' prosocial motivation as an intermediary variable based on social cognitive theory and social learning theory, and explores the influence mechanism of the relationship between leader's harmonious orientation and employees' innovation behavior. Through the questionnaire survey, the following conclusions are obtained: the harmonious orientation of leadership will significantly affect the innovative behavior of employees, which is manifested in that the leader's sincere harmony orientation significantly positively affects the employees' innovation behavior; the leader's superficial harmony orientation significantly negatively affects the innovative behavior of employees. Employees' prosocial motivation plays a mediating role between leader's harmonious orientation and employees' innovation behavior.
\end{abstract}

Keywords: Leader's Harmonious orientation, Employees' Prosocial Motivation, Employees' innovation behavior

\section{INTRODUCTION}

In the wave of innovation-driven development, innovation has become a sharp weapon of enterprise transformation and development and attracted much attention. As the power source of enterprise innovation ${ }^{[i]}$, employees' innovation behavior will directly determine the efficiency and achievement of enterprise innovation, so how to stimulate employees' innovation behavior has become a hot topic.

Innovation depends on a certain cultural atmosphere, and there are significant differences between eastern and western cultures ${ }^{[i]}$. The values formed by different social cultures will directly affect the form and rhythm of innovation. Therefore, the study of the innovation needs of Chinese Enterprises employees and the local cultural background fit, as the essence of China's excellent traditional culture, harmony on the Chinese People's thinking and behavior far-reaching ${ }^{[i i i]}$. In view of this, it is of theoretical significance and research value to explore the relationship between harmony orientation and employees' innovation behavior in the context of local culture.

Research shows that the cultural value orientation of leaders tends to have a profound impact on employees' work behaviors ${ }^{[i v]}$. Through the observation of leadership behavior, employees will know whether leader's value and support innovation, whether they are willing to take the responsibility and risk of innovation, so that employees can adjust their innovation behavior. Based on social learning theory, leadership harmony orientation will have a far-reaching impact on employees' prosocial motivation. A leader with a seemingly harmonious orientation attaches more importance to the maintenance of face and relationships, creating the illusion of unity and harmony; on the contrary, a leader with a sincere and harmonious orientation will give employees resources and opportunities for development and actively deal with potential conflicts, create a truth-seeking atmosphere for your employees. Employees' willingness to help others in a context will vary, as will the resources they need to innovate. In view of this, the purpose of this study is to 
select employees' prosocial motivation as an intermediary variable, and to explore whether it will play an intermediary role between leadership harmony orientation and employees' innovation behavior.

To sum up, the purpose of this study is to explore the mechanism of the influence of harmonious leadership orientation on employees' innovation behavior in the context of local culture, and to introduce the mediating mechanism in the process of the influence of employees' prosocial motivation, push the enterprise to push the employees' innovation behavior from the angle of cultural value.

\section{THEORETICAL REVIEW AND RESEARCH HYPOTHESIS}

\subsection{Variable Definition}

\subsubsection{Leader's Harmonious Orientation}

The idea of harmony can be traced back to the Western Zhou dynasty. Confucius advocated the gentleman and different, villain and different ${ }^{[\mathrm{v}]}$. In the field of organizational management, Leung defines harmonious orientation as an individual's spiritual pursuit and personal practice of high-order virtue and character. At present, scholars generally agree with Leung's definition of harmony orientation ${ }^{[\mathrm{vi}]}$. Huang Guangguo divides harmony orientation into superficial harmony and sincere harmony. The former refers to the contradiction and conflict under the surface of harmony, while the latter refers to the harmony of heart and mouth. Therefore, based on the previous research, this paper turns the leadership harmony orientation into the leadership sincere harmony orientation and the leadership superficial harmony orientation, the former is that the leadership pursues the team long-term development, and encourages the collision and exchange of different viewpoints, the latter is that the leader pursues his team's short-term benefit, and deliberately conceals a value orientation of the team's contradiction.

\subsubsection{Employees' Prosocial Motivation}

Batson first defined prosocial motivation, pointing out that prosocial motivation is the employees' willingness to give and benefit to others ${ }^{\text {[vii] }}$. Grant then points out that prosocial motivation is the idea that employees act out of concern for helping and contributing to others ${ }^{[\text {viii }]}$. Zhao Dexian and others pointed out that pro-social motivation is the desire of employees to help others in the workplace ${ }^{[\mathrm{ix}]}$. Combing the above literature, we can see that the definition of Batson has higher authority and is widely used by scholars at home and abroad. Therefore, this paper uses Batson's research for reference, employees' prosocial motivation is defined as employees' willingness to contribute to and benefit others (leaders, customers, etc.).

\subsubsection{Employees' Innovation Behavior}

For example, Scott \& Bruce think that employees' innovation behavior includes identifying problems, generating innovative ideas, and finding solutions, finally, the idea of innovation will be "Product" and "Institutionalized ${ }^{[\mathrm{x}]}$. Amabile points out that employees' innovation involves the generation and successful implementation of creative ideas ${ }^{[x i]}$. Zhou \& George believe that employees' innovation behavior includes the creation of innovative ideas, content, promotion and development of executive programs ${ }^{[x i i]}$. Liao Bing and others define employees' innovation behavior as the behavior that employees produce novel and valuable ideas or solutions and implement them in their work ${ }^{[\mathrm{xiii}]}$. From the perspective of process, scholars at home and abroad generally take into account both the generation and execution of innovative ideas. Therefore, this paper intends to follow this definition method, employees' innovation behavior is defined as the behavior that employees' generate innovative ideas and put them into practice in their work.

\subsection{Research Hypothesis}

\subsubsection{Leader's Harmonious Orientation and Employees' Innovation Behavior}

According to Bandura's theory of social learning, individuals can acquire new behavior patterns through direct experience or observation of others' attitudes, values and behaviors. Given that the values of leadership are the embodiment of group norms, employees observe and learn from leaders at work and exhibit behaviors similar to those of leaders. Seemingly harmonious leaders focus on short-term benefits, hide potential conflicts within the organization, and create the illusion of unity and harmony. Under its influence, employees are more reluctant to exchange views with others for their own safety, and knowledge sharing within the organization is restricted $^{[\text {xiv }]}$, which is not conducive to stimulating employees' innovation behavior. Sincere and harmonious leaders focus on the long-term development of the organization, promote the exchange of different views, and actively build an organizational atmosphere of openness and exchange. Under the influence of sincere and harmonious leadership, employees will open up to others and actively exchange different views. Some studies have shown that knowledge sharing can stimulate employees' innovation behavior ${ }^{[\mathrm{xv}]}$. In conclusion, make the following assumptions:

H1: The leader's harmony orientation can significantly influence employees' innovation behavior. 
H1a: The leader's superficial harmony orientation negatively affects employees' innovation behavior.

H1b: The leader's sincere harmony orientation positively affects employees' innovation behavior.

\subsubsection{The Intermediary Effect of Employees' Prosocial Motivation}

According to the social cognitive theory, the external environment is one of the key factors that affect the employees' behavior and subjectivity. According to this reasoning, the leaders of the superficial harmony orientation often adopt the evasive attitude to the conflicts in the organization, pay more attention to the maintenance of face and relations, and create the illusion of unity and harmony. Therefore, in a seemingly harmonious leadership environment, employees under the pressure of leadership for self-preservation, it is difficult to produce the will to help others, prosocial motivation weakened. On the contrary, sincere and harmonious leadership shows concern for the long-term development of employees, gives employees the resources and opportunities for development and actively deals with potential contradictions, creating an atmosphere for employees to pursue the truth. In this situation, employees feel the kindness and care from their leaders, which will lead to the idea of contributing to the organization and stimulate their own prosocial motivation. This leads to the following assumptions:

$\mathrm{H} 2$ : The leader's harmony orientation significantly affects employees' prosocial motivation.

H2a: The leader's superficial harmony orientation negatively affects employees' prosocial motivation.

$\mathrm{H} 2 \mathrm{~b}$ : The leader's sincere harmony orientation positively affects employees' prosocial motivation.

According to the theory of planned behavior, attitude and motivation are the most direct factors that influence individual behavior. According to this reasoning, employees' prosocial motivation stimulates the creation of ideas ${ }^{[x v i]}$, thus promoting innovative behavior. On the one hand, employees' prosocial motivation show more empathy and empathy towards others, and get new ideas in the process of helping others to solve problems, which is beneficial to employees' innovation behavior. On the other hand, the positive emotion brought by prosocial motivation is the key factor for the formation of innovation behavior, which can improve the employees' open thinking, contribute to the emergence of their innovative ideas ${ }^{[\mathrm{xvii}]}$, and then promote their innovative behavior. To sum up, the following assumptions are made:

H3: The employees' prosocial motivation positively influences employees' innovation behavior.

Based on the hypothesis of the relationship between the leader's harmonious orientation and the employees' innovation behavior, the leader's harmonious orientation will affect the employees' innovation behavior indirectly through the intermediary mechanism of the employees' prosocial motivation. So make the following assumptions:

H4: The employees' prosocial motivation plays a partial or complete mediating role in the influence of leader's harmony orientation on employees' innovation behavior.

Based on the above research assumptions, the theoretical model is shown in Figure 1.

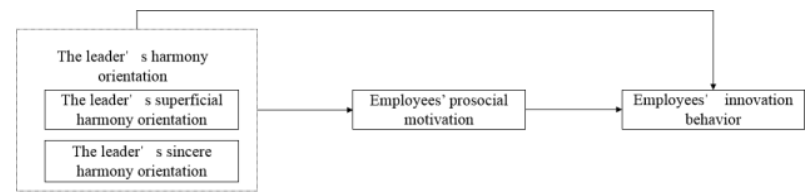

Figure 1. Theoretical model

\section{RESEARCH DESIGN}

\subsection{Scale Design}

The Leader's Harmony Orientation Scale, which was developed by Chen et $\mathrm{al}^{[\mathrm{xviii}]}$, is a mature scale widely accepted and used by the academic circles. The prosocial motivation scale was derived from the maturity scale developed by Grant \& Sumanth ${ }^{[\mathrm{xix}]}$. The employees' innovation behavior scale, derived from Scott \& Bruce's research $^{[\mathrm{xx}]}$, has been widely accepted by the academic community and has been proved to be applicable to the organizational context in China.

\subsection{Data Collection}

This research carries on the data survey through the online and the offline two ways, altogether issues and reclaims 330 questionnaires. In order to ensure the validity of the questionnaire, 290 questionnaires were obtained after eliminating the invalid questionnaire, and the effective recovery rate was $87.88 \%$. A descriptive statistical analysis of the sample is shown in Table $\mathbf{1 .}$

Table 1 Descriptive analysis of demographics variables

\begin{tabular}{|c|c|c|c|}
\hline Statistics & Classification & Frequency & Percentage \% \\
\hline \multirow{3}{*}{ Gender } & male & 166 & 57.2 \\
\hline & female & 124 & 42.8 \\
\hline & 18 25years old & 48 & 16.6 \\
\hline Age & $\begin{array}{l}\text { 26 30years old } \\
31 \sim 35 \text { years old } \\
35 \sim 40 \text { years old }\end{array}$ & $\begin{array}{l}111 \\
90 \\
31\end{array}$ & $\begin{array}{l}38.3 \\
31.0 \\
10.7\end{array}$ \\
\hline
\end{tabular}




\begin{tabular}{cccc} 
& 40 years old and above & & 3.4 \\
& below speciailist & 10 & 3.8 \\
& specialist & 11 & 21.0 \\
Education level & undergraduate & 61 & 48.6 \\
& master degree and above & 141 & 2.6 \\
under 2 years & 77 & 21.7 \\
Working years & $3-5$ years & 63 & 13.1 \\
& $6-10$ years & 38 & 37.6 \\
\hline
\end{tabular}

\section{DATA ANALYSIS AND HYPOTHESIS TESTING}

\subsection{Data Quality Analysis}

First of all, the Cronbach's $\alpha$ coefficients of each variable were $0.933,0.844,0.892$ and 0.909 . The reliability of the variables was very good. Secondly, principal component analysis extracted four common factors, explained the total variance of $72.151 \%$, showed that the four factors selected have good representation. At last, Pearson correlation coefficient was used to analyse the relationship between leadership superficial harmony orientation ( $\mathrm{r}=-0.164, \mathrm{p}<0.05)$ and employees' innovation behavior, and leader's sincere harmony orientation $(\mathrm{r}=0.645, \mathrm{p}<0.01)$ was positively correlated with employees' innovation behavior. The employees' prosocial motivation $(r=0.649, p<0.01)$ was positively correlated with employees' innovation behavior, while the leader's superficial harmony orientation $(r=-0.183, p$ $<0.01$ ) was negatively correlated with employees' prosocial motivation. The leader's sincere harmony orientation $(r=0.638, p<0.01)$ was positively correlated with employees' prosocial motivation.

\subsection{Hypothetical Test}

The first step, the main effect test: as shown in Table 2 , the leader's superficial harmony orientation $(\beta=$ $0.108, \mathrm{p}<0.05)$ had a significant negative effect on employees' innovation behavior, and H1a was tested, as shown in Table 2, M6, the leader's sincere harmony orientation $(\beta=0.643, \mathrm{p}<0.001)$ had significant positive effect on employees' innovation behavior, which was verified by H1b. Population $\mathrm{H} 1$ is tested.

The second step, the mediating effect test: as shown in Table 2, the leader's superficial harmony orientation ( $\beta$ $=-0.116, p<0.05)$ has a significant negative effect on employees' prosocial motivation, and $\mathrm{H} 2 \mathrm{a}$ is verified as shown in Table 3, M3, the leader's sincere harmony orientation $(\beta=0.631, p<0.001)$ had significant positive effect on employees' prosocial motivation, and $\mathrm{H} 2 \mathrm{~b}$ was verified. The whole of $\mathrm{H} 2$ is verified. As M7 in Table 2 shows, the employees' prosocial motivation $(\beta=0.651, \mathrm{p}$ $<0.001$ ) has a significant positive effect on employees' innovation behavior. As shown in M8 of Table 2, when both leader's superficial harmony orientation and employees' prosocial motivation were included in the equation, the independent variable leader's superficial harmony orientation for the dependent variable innovation coefficient increased from -0.108 to -0.063 , it shows that employees' prosocial motivation plays a significant and complete mediating role between leader's superficial harmony and employees' innovation behaviors; as M9 in Table 2 shows, when both leader's sincere harmony orientation and employees' prosocial motivation are included in the equation, the innovation coefficient of independent-variable leader's sincere harmony orientation decreased from 0.643 to 0.400 , indicating that employee's prosocial motivation played a part of mediating role between leader's sincere harmony and employees' innovation behaviors. Population H4 is verified.

Table 2 Analysis of the mediating effect of prosocial motivation

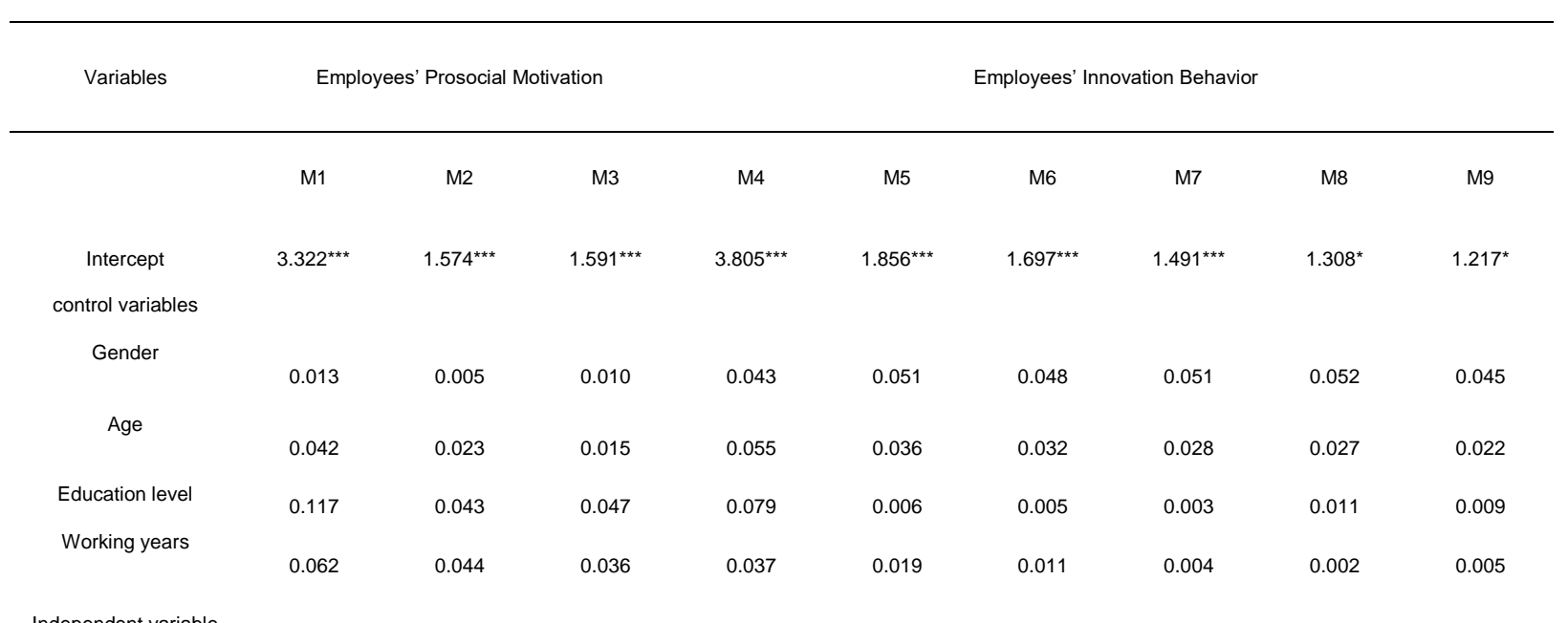




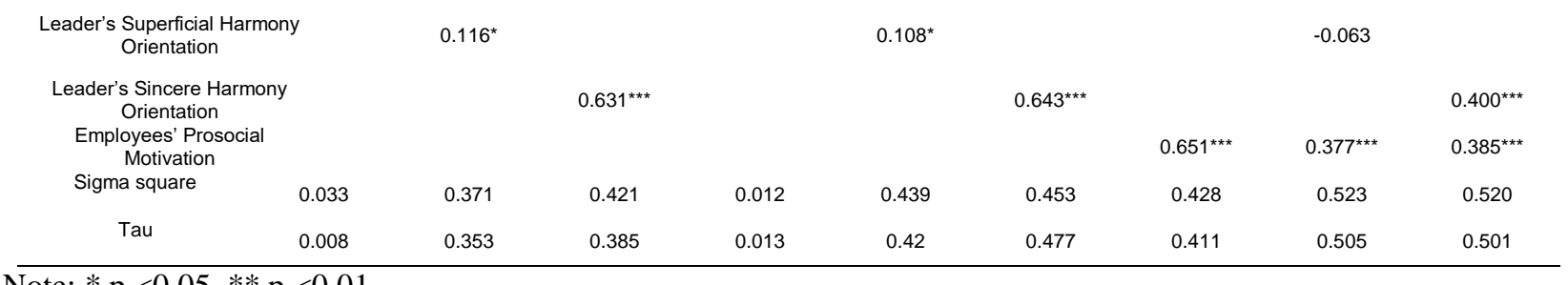

\section{RESEARCH CONCLUSION}

The leader's sincere harmony orientation has significant positive influence on employees' innovation behaviors, and the leader's superficial harmonious orientation has significant negative influence on employees' innovation behaviors. This shows that the leader's sincere harmony orientation is actively concerned about the future long-term development of employees, conducive to stimulating innovative behaviors of employees. On the other hand, a seemingly leader's harmony orientation will create a negative organizational climate, which is not conducive to stimulating employees' innovation behaviors.

Employees' prosocial motivation plays a mediating role in the influence mechanism of leader's harmony orientation on employee innovation behaviors. This shows that under the direction of leader's sincere harmony, employees receive support and encouragement from the leadership, the motivation of information transmission is higher, employees' social motivation is enhanced, and it is easier to transposition thinking to promote their innovation behaviors. Under the leader's surface orientation, in order to maintain face and avoid conflict, employees are difficult to produce the willingness to contribute to others, the employee's prosocial motivation is weakened, which is not conducive to stimulating their innovation behaviors.

\section{REFERENCES}

[1] Du Jing, Mu Huina, Liu Yiting. Does collectivism really hinder innovation?-An empirical study based on the effect of situation[J]. Studies in Science of Science, 2014, 32(06): 919-926.

[2] Sui Yang, Chen Yunyun, Wang Hui. Innovation atmosphere, innovation efficacy and team innovation: The moderating effect of team leadership[J]. Journal of Psychology, 2012(2): 237 -248 .

[3] Wang Yunmei, Zhou Hongmei. The concept of harmony in Chinese traditional culture and the construction of modern corporate culture $[\mathrm{J}]$. Hunan Social Sciences, 2010(6): 106-108.

[4] Yang Xianchuan, Zhang Lei. The golden mean value orientation and employees' innovative behavior - a moderated intermediary model[J]. Technology Economics and Management Research, 2018(02):54-58
[5] Jia Leilei, Pan Yuan. The core values of Chinese traditional culture: Harmony, benevolence and nature $[\mathrm{J}]$. Journal of Southeast University (Philosophy and Social Sciences Edition), 2012, 14(03): 56-60+127.

[6] Leung K, Koch P T, Lu L. A Dualistic Model of Harmony and its implications for conflict management in Asia[J]. Asia Pacific Journal of Management, 2002, 19(2-3): 201-220.

[7] Batson C D. Prosocial motivation: Is it ever truly altruistic?[J]. Advances in Experimental Social Psychology, 1987, 20: 65-122.

[8] Grant A M. Relational job design and the motivation to make a prosocial difference[J]. Academy of Management Review, 2007, 32(2): 393-417.

[9] Zhao Dexian, Zheng Xingshan. Prosocial Motivation at Work: Research summary and prospects[J]. China Human Resources Development, 2016 (09): 36$43+73$.

[10] Scott S G, Bruce R A. Determinants of innovative behavior: A path model of individual innovation in the workplace $[\mathrm{J}]$. Academy of Management Journal, 1994, 37(3):580-607.

[11] Amabile, Teresa M. Assessing the work environment for creativity $[\mathrm{J}]$. Academy of management Journal, 1996, 39(5): 1154-1184.

[12] Zhou J, George J M. When job dissatisfaction leads to creativity: Encouraging the expression of voice[J]. Academy of Management Journal, 2001, 44(4): 682696.

[13] Liao Bing, Dong Wenqiang. A Study on the relationship between knowledge workers' moderate thinking, organizational harmony and individual innovation behavior[J]. Science \& Technology Progress and Policy, 2015, 32(07): 150-154.

[14] Wei Xin, Zhang Zhixue. Why is there a lack of inhibitory comments in organizations?[J]. Management World, 2010(10): 99-109+121.

[15] Cao Yong, Xiang Yang. Corporate knowledge governance, knowledge sharing, and employees' innovative behavior: The mediating effect of social capital and the moderating effect of absorptive capacity[J]. Studies in Science of Science, 2014, 32(01): 92-102.

[16] Hoever I J, Van Knippenberg D, Van Ginkel W P, et al. Fostering team creativity: Perspective taking as key to unlocking diversity's potential[J]. Journal of Applied Psychology, 2012, 97(5): 982-996. 
[17] Amabile T M. A model of creativity and innovation in organizations[J]. Research in Organizational Behavior, 1988, 10: 129-131.

[18] Chen X P, Peng S Q. Guanxi dynamics: Shifts in the closeness of ties between Chinese co-workers[J]. Management and Organization Review, 2008, 4(1): 63-80.

[19] Grant A M, Sumanth J J. Mission possible? The performance of prosocially motivated employees depends on manager trustworthiness[J]. Journal of Applied Psychology, 2009, 94(4): 927-944.

[20] Scott S G, Bruce R A. Determinants of innovative behavior: A path model of individual innovation in the workplace[J]. Academy of Management Journal, 1994, 37(3): 580-607. 